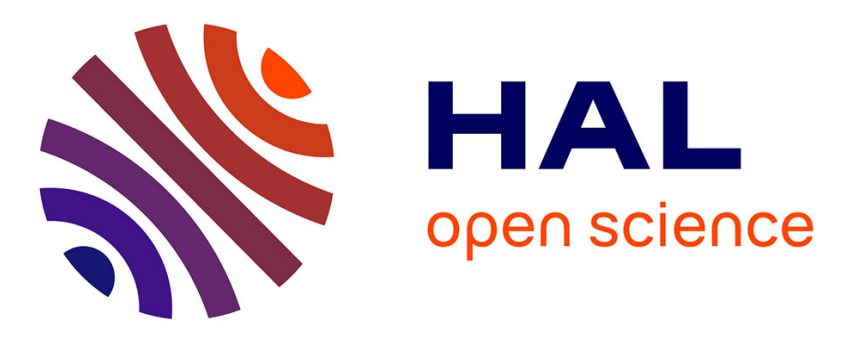

\title{
Abnormal pulmonary endothelial cells may underlie the enigmatic pathogenesis of chronic thromboembolic pulmonary hypertension
}

Olaf Mercier, Jennifer Arthur Ataam, Nathaniel Langer, Peter Dorfmüller, Lilia Lamrani, Florence Lecerf, Benoit Decante, Philippe Dartevelle, Saadia Eddahibi, Elie Fadel

\section{To cite this version:}

Olaf Mercier, Jennifer Arthur Ataam, Nathaniel Langer, Peter Dorfmüller, Lilia Lamrani, et al.. Abnormal pulmonary endothelial cells may underlie the enigmatic pathogenesis of chronic thromboembolic pulmonary hypertension. The Journal of Heart and Lung Transplantation, 2017, 36 (3), pp.305 - 314. 10.1016/j.healun.2016.08.012 . hal-01824263

\section{HAL Id: hal-01824263 \\ https://hal.umontpellier.fr/hal-01824263}

Submitted on 17 Dec 2019

HAL is a multi-disciplinary open access archive for the deposit and dissemination of scientific research documents, whether they are published or not. The documents may come from teaching and research institutions in France or abroad, or from public or private research centers.
L'archive ouverte pluridisciplinaire HAL, est destinée au dépôt et à la diffusion de documents scientifiques de niveau recherche, publiés ou non, émanant des établissements d'enseignement et de recherche français ou étrangers, des laboratoires publics ou privés. 


\title{
Abnormal pulmonary endothelial cells may underlie the enigmatic pathogenesis of chronic thromboembolic pulmonary hypertension
}

\author{
Olaf Mercier, MD, PhD, ${ }^{a, b, 1}$ Jennifer Arthur Ataam, MSc, ${ }^{a, 1}$ \\ Nathaniel B. Langer, MD, ${ }^{a, b}$ Peter Dorfmüller, MD, PhD, ${ }^{a, c}$ Lilia Lamrani, MSc, ${ }^{a}$ \\ Florence Lecerf, MSc, ${ }^{a}$ Benoit Decante, $\mathrm{MSc}^{,}{ }^{a}$ Philippe Dartevelle, MD, ${ }^{\mathrm{a}, \mathrm{b}}$ \\ Saadia Eddahibi, PhD, a,d and Elie Fadel, MD, PhD ${ }^{\mathrm{a}, \mathrm{b}}$
}

\begin{abstract}
From the ${ }^{a}$ Research and Innovation Unit, INSERM U999, DHU TORINO, Paris Sud University, Marie Lannelongue Hospital, Le Plessis Robinson, France; ${ }^{b}$ Departments of Thoracic and Vascular Surgery and Heart-Lung

Transplantation, Marie Lannelongue Hospital, Le Plessis Robinson, France; ${ }^{c}$ Pathology, Marie Lannelongue Hospital, Le Plessis Robinson, France; and the ${ }^{d} I N S E R M$ U1046, CNRS UMR 9214, Université de Montpellier, CHU Arnaud de Villeneuve Montpellier, Montpellier, France.
\end{abstract}

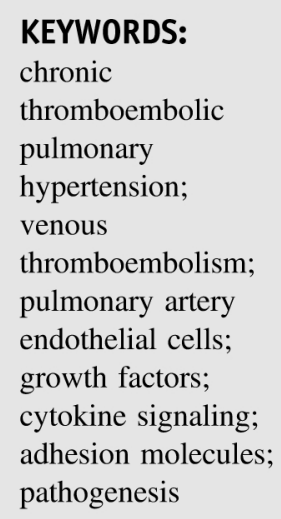

\begin{abstract}
BACKGROUND: Chronic thromboembolic pulmonary hypertension results from chronic mechanical obstruction of the pulmonary arteries after acute venous thromboembolism. However, the mechanisms that result in the progression from unresolved thrombus to fibrotic vascular remodeling are unknown. We hypothesized that pulmonary artery endothelial cells contribute to this phenomenon via paracrine growth factor and cytokine signaling.

METHODS: Using enzyme-linked immunosorbent assay and cell migration assays, we investigated the circulating growth factors and cytokines of chronic thromboembolic pulmonary hypertension patients as well as the cross talk between pulmonary endothelial cells and pulmonary artery smooth muscle cells and monocytes from patients with chronic thromboembolic pulmonary hypertension in vitro.

RESULTS: Culture medium from the pulmonary endothelial cells of chronic thromboembolic pulmonary hypertension patients contained higher levels of growth factors (fibroblast growth factor 2), inflammatory cytokines (interleukin $1 \beta$, interleukin 6 , monocyte chemoattractant protein 1 ), and cell adhesion molecules (vascular cell adhesion molecule 1 and intercellular adhesion molecule 1). Furthermore, exposure to the culture medium of pulmonary endothelial cells from patients with chronic thromboembolic pulmonary hypertension elicited marked pulmonary artery smooth muscle cell growth and monocyte migration.

CONCLUSIONS: These findings implicate pulmonary endothelial cells as key regulators of pulmonary artery smooth muscle cell and monocyte behavior in chronic thromboembolic pulmonary hypertension and suggest a potential mechanism for the progression from unresolved thrombus to fibrotic vascular remodeling.
\end{abstract}

${ }^{1}$ O.M. and J.A.A. contributed equally to this study.

Reprint requests: Olaf Mercier, MD, PhD, Research and Innovation Unit, Department of Thoracic and Vascular Surgery and Heart-Lung Transplantation, Marie Lannelongue Hospital, 133 Ave de la Resistance, 92350 Le Plessis Robinson, France. Telephone: +33-140-948-695.

E-mail address: o.mercier@ccml.fr
Chronic thromboembolic pulmonary hypertension (CTEPH) is a rare disease resulting from progressive mechanical obstruction of the pulmonary arteries. Surgical pulmonary endarterectomy (PEA) is the standard of care, without which patients develop progressive, lethal right-sided heart 
failure. ${ }^{1}$ Although the precise incidence remains unknown, it is estimated that CTEPH will develop in $0.1 \%$ to $9.1 \%$ of patients who suffer an acute pulmonary embolism. ${ }^{2}$ Although prior studies have found that risk factors for venous thromboembolism do not increase the risk of CTEPH, $74.8 \%$ of patients in the European CTEPH Registry have evidence of prior pulmonary embolism and $56.1 \%$ of prior deep vein thrombosis. ${ }^{3,4}$ Subsequently, processes other than venous thromboembolism are thought to contribute to the overall pathogenesis. Despite ongoing research, these processes remain poorly understood.

Given the close anatomic relationship between the pulmonary artery endothelium and the thrombus, it is possible that CTEPH is in part a disease of pulmonary endothelial cells (PECs). Endothelial cell dysfunction is a known contributor to the pulmonary vascular remodeling seen in idiopathic pulmonary arterial hypertension (PAH), possibly via abnormal growth factor and/or cytokine synthesis and secretion. Studies in rodent models of lowflow inferior vena cava thrombosis have shown that clot resolution occurs by inflammatory cell-dependent recanalization and that stimulation or inhibition of the inflammatory process affects overall thrombus evolution. ${ }^{5-8}$

Given their interaction with the clot, and evidence from studies of other types of pulmonary hypertension, we hypothesized that PECs play a central role in the pathogenesis of CTEPH. Here, we used PECs from PEA specimens to investigate the cross talk between the pulmonary artery endothelium and both pulmonary artery smooth muscle cells (PASMCs) and monocytes.

\section{Methods}

This study was conducted in accordance with the amended Declaration of Helsinki and approved by the Marie Lannelongue Hospital Institutional Review Board. Written, informed consent was obtained from all patients.

\section{Study population and plasma analysis}

PEA specimens from 109 CTEPH patients and pulmonary artery specimens from 94 control patients undergoing lobectomy or pneumonectomy for localized lung cancer were collected. Pulmonary arteries were harvested as far as possible from the tumor. Blood samples were collected from both groups during the pre-operative evaluation. Pre-operative transthoracic echocardiography confirmed the absence of pulmonary hypertension (right ventricular systolic pressure $<30 \mathrm{~mm} \mathrm{Hg}$ ) in all control patients. The clinical characteristics of both groups are summarized in Table 1.

Plasma levels of inflammatory cytokines (monocyte chemoattractant protein 1 [MCP-1], interleukin $1 \beta \quad[\mathrm{IL}-1 \beta]$, and interleukin 6 [IL-6]), adhesion molecules (intercellular adhesion molecule 1 [ICAM-1] and vascular cell adhesion molecule 1 [VCAM-1]), and growth factors (epidermal growth factor [EGF], platelet-derived growth factor [PDGF], fibroblast growth factor 2 [FGF-2]), and transforming growth factor- $\beta 1$ [TGF- $\beta 1]$ ) were measured using a sandwich enzyme-linked immunosorbent assay (ELISA) Quantikine ELISA kit (R\&D Systems, Minneapolis, $M N$ ), according to the manufacturer's instructions.

\section{Cell isolation, culture, and characterization}

PECs were isolated by enzymatic digestion of PEA specimens or pulmonary arteries from a randomly generated sub-set of 20 patients and cultured as described. ${ }^{9}$ Baseline characteristics of the included patients are summarized in Table 2. Endothelial cells were defined as CD31-positive and desmin/vimentin-negative and constituted $>95 \%$ of culture cells (Figure 1). All cells used in the study were between passages 3 and 6 . Briefly, human PECs were obtained by Dispase I digestion (Roche Diagnostics, Penzbeg, Germany) of PEA specimens (CTEPH) and a $5-\mathrm{cm}^{3}$ pulmonary artery fragment (control) left at $37^{\circ} \mathrm{C}$ overnight. The suspension was filtered, plated onto $0.1 \%$ gelatin-coated wells, and grown in MCDB131 medium (Invitrogen, Cergy-Pontoise, France) supplemented with $10 \%$ fetal calf serum (FCS), $50 \mathrm{U} / \mathrm{ml}$ of penicillin/streptomycin, $4 \mathrm{mmol} / \mathrm{L} \mathrm{L}$-glutamine, $25 \mathrm{mmol} / \mathrm{L} 4-(2-$ hydroxyethyl)-1-piperazineethanesulfonic acid (HEPES), $10 \mathrm{U} / \mathrm{ml}$ heparin, $1 \mu \mathrm{g} / \mathrm{ml}$ human endothelial cell growth supplement, and $10 \mathrm{ng} / \mathrm{ml}$ vascular endothelial growth factor (VEGF; Promocell, Heidelberg, Germany). Immunomagnetic purification of PECs was then performed with anti-platelet endothelial cell adhesion molecule 1 (cluster of differentiation [CD] 31) monoclonal antibodylabeled DynaBeads (Dynal; Biotech, Compiègne, France).

To characterize the endothelial cell phenotype, cells were labeled with acetylated low-density lipoprotein (LDL) coupled to a fluorescent carbocyanine dye (DiI-Ac-LDL; Tebu, Le Perray en Yvelines, France) and stained with antibodies against the

Table 1 Baseline Characteristics of Patient Groups for Plasma Analysis

\begin{tabular}{lccc}
\hline & \multicolumn{1}{c}{ Control } & \multicolumn{1}{c}{ CTEPH } \\
\cline { 2 - 4 } Variable $^{\mathrm{a}}$ & $(n=94)$ & $(n=109)$ & $p$-value \\
\hline Age, years $_{\text {Sex }}$ & $62.6 \pm 12.2$ & $60.23 \pm 14.4$ & 0.22 \\
$\quad$ Male & $58(62)$ & $61(56)$ & \\
$\quad$ Female & $37(38)$ & $48(44)$ & 0.46 \\
Smoker & $64(71)$ & $40(37)$ & 0.001 \\
Mean pulmonary artery pressure, $\mathrm{mm} \mathrm{Hg}$ & $17.7 \pm 3.5$ & $46.4 \pm 11.0$ & 0.001 \\
Cardiac index, liters/min/m & - & $2.6 \pm 0.7$ & - \\
Total pulmonary resistance, dynes/sec/cm & & & \\
\hline
\end{tabular}




\begin{tabular}{lccc}
\hline & \multicolumn{2}{c}{ Control } & CTEPH \\
\cline { 2 - 4 } Variable $^{\mathrm{a}}$ & $(n=10)$ & $(n=10)$ & $p$-value \\
\hline Age, years & $59.8 \pm 12.0$ & $66.6 \pm 10.2$ & 0.19 \\
Sex & & & 0.83 \\
$\quad$ Male & $3(30)$ & $5(50)$ & \\
$\quad$ Female & $7(70)$ & $5(50)$ & 0.28 \\
Smoker & $5(50)$ & $3(30)$ & 0.001 \\
Mean pulmonary artery pressure, $\mathrm{mm} \mathrm{Hg}$ & $19.6 \pm 3.7$ & $51.4 \pm 12.1$ & - \\
Cardiac index, liters/min/m & - & $2.62 \pm 0.6$ & - \\
Total pulmonary resistance, dynes/sec/ $/ \mathrm{cm}^{-5}$ & - & $936.5 \pm 294.8$ & \\
\hline
\end{tabular}

CETPH, chronic thromboembolic pulmonary hypertension.

${ }^{a}$ Continuous variables are presented as mean \pm standard deviation and categoric variables as number $(\%)$.

endothelial cell-specific lectin Ulex europaeus agglutinin-1 (UEA-1; Sigma-Aldrich, Lyon, France) and CD31 (Dako; Figure 1). CTEPH endothelial cells $(n=3)$ were also characterized using flow cytometry, Western blot, and quantitative real-time polymerase chain reaction and compared with commercial human pulmonary artery endothelial cells ( $n=3$; Lonza). For Western blot, cells were homogenized and sonicated in phosphate-buffered saline (PBS) containing protease inhibitors, and $20 \mu \mathrm{g}$ of proteins were used to quantify CD31, vascular endothelial-cadherin (CD144), von Willebrand factor (vWF), VEGF receptor 2 (VEGF-R2), p120-catenin (Abcam), and $\beta$-actin (Sigma-Aldrich, Saint-Quentin Fallavier, France). Messenger RNA (mRNA) levels of expression of CD31, VE-cadherin, vWF, VEGF-R2, and p120-catenin (TaqMan, Applied Biosystems) were assessed by real-time quantitative polymerase chain reaction. Relative quantification was calculated by normalizing the $\mathrm{Ct}$ (threshold cycle) of the gene of interest to the $\mathrm{Ct}$ of $18 \mathrm{~S}$ in the same sample according to the comparative $\mathrm{Ct}$ method ( $\Delta \Delta \mathrm{Ct}$ method).

Endothelial cells were labeled with fluorophore-conjugated monoclonal anti-CD31 (Sigma-Aldrich) and fluorophore conjugated anti-vascular endothelial-cadherin (eBiosciences). Flow cytometry gating conditions and mean fluorescence intensity were set and normalized against isotype- and fluorophore-matched nonimmune immunoglobulin $\mathrm{G}$, respectively. Flow cytometry results were acquired with a MACSQuant flow cytometer (Miltenyi Biotec, Paris, France) and analyzed by FlowJo software (Tree Star, Inc., Ashland, OR). Our results confirmed that isolated endothelial cells from CTEPH patients were human PECs (Figure 1).

PASMCs were isolated from the same specimens as PEC and cultured as previously described. ${ }^{9}$ To ensure that isolated cells were PASMCs, cells from each culture were assessed for expression of smooth muscle $\alpha$-actin by immunostaining, as previously described $^{9}$ (Figure 1). Briefly, human PASMCs were cultured from explants. Pulmonary arteries or PEA specimens (diameter of 5-10 mm) were kept in Dulbecco's Modified Eagle's medium (DMEM) at $4^{\circ} \mathrm{C}$ before their intimal cell layer and residual adventitial tissue were stripped off using forceps. The dissected media of the vessels was then cut into small pieces $(3-5 \mathrm{~mm}$ ), which were transferred into cell culture flasks. To allow the PASMCs to grow out, the vessel tissues were incubated in DMEM supplemented with $20 \% \mathrm{FCS}, 2 \mathrm{mmol} /$ liter L-glutamine, and antibiotics ( $100 \mathrm{U} / \mathrm{ml}$ penicillin and $0.1 \mathrm{mg} / \mathrm{ml}$ streptomycin). After 2 weeks of incubation, the PASMCs collected in the culture medium and the vessel tissues were transferred into new cell culture flasks. SMCs were defined as $\alpha$-actin-positive and constituted $>95 \%$ of culture cells (Figure 1). Cultured PASMCs were used between passages 1 and 3. Explants from the same pulmonary artery were usually transferred several times.
Monocytes were isolated from venous blood samples using Ficoll-Paque (Amersham Biosciences, Orsay, France), followed by Percoll density gradient centrifugation, as previously described. ${ }^{10}$

\section{Effects of PEC medium}

The levels of growth factors (PDGF, FGF-2, EGF, TGF- $\beta 1$ ), inflammatory cytokines (MCP1, IL1- $\beta$, IL-6), and cell adhesion molecules (ICAM-1, VCAM-1) in the PEC medium were measured by ELISA (R\&D Systems) according to the manufacturer's instructions.

PECs were seeded in 24-well plates containing MCDB131 medium supplemented with $10 \%$ FCS at a density of $5 \times 10^{4}$ cells/well and allowed to adhere and grow for 24 hours. These cells were then cultured in growth factor-free MCDB131 medium for 24 hours, after which the medium was collected and used as the PEC medium.

PASMCs were cultured in DMEM supplemented with $15 \%$ FCS, seeded in 24-well plates at a density of $5 \times 10^{4}$ cells per well, and allowed to adhere. Cells were synchronized by 48 hours of growth arrest in DMEM without FCS, after which $1 \mathrm{ml}$ of PEC medium or $5 \%$ FCS was added. Cells were incubated for 24 hours with $\left[{ }^{3} \mathrm{H}\right]$ thymidine $(1 \mu \mathrm{Ci} / \mathrm{ml})$, washed twice with PBS, treated with ice-cold $10 \%$ trichloroacetic acid, and dissolved in $0.1 \mathrm{~N}$ $\mathrm{NaOH}(0.5 \mathrm{ml} /$ well). Incorporated radioactivity was quantified and reported as cpm/well.

Monocyte chemotaxis was quantified, alone and in the presence of an MCP-1-blocking antibody, as previously described. $^{10}$

\section{Immunofluorescence and histochemical staining}

Paraffin-embedded sections ( $5 \mu \mathrm{m}$ thick) of PEA specimens were mounted on Superfrost Plus slides (Fisher Scientific). Slides were dewaxed in $100 \%$ toluene and rehydrated by immersion in decreasing ethanol concentrations (100\%, $95 \%$, and $70 \%$ ), followed by distilled water. The sections were blocked with PBS containing $1 \%$ (weight/volume) bovine serum albumin and $0.1 \%$ (volume/volume) Tween 20 and were incubated at $4{ }^{\circ} \mathrm{C}$ overnight with an anti-human vWF monoclonal antibody to detect endothelial cells (1:250; Santa Cruz Biotechnology), an anti-human $\alpha$-actin antibody 

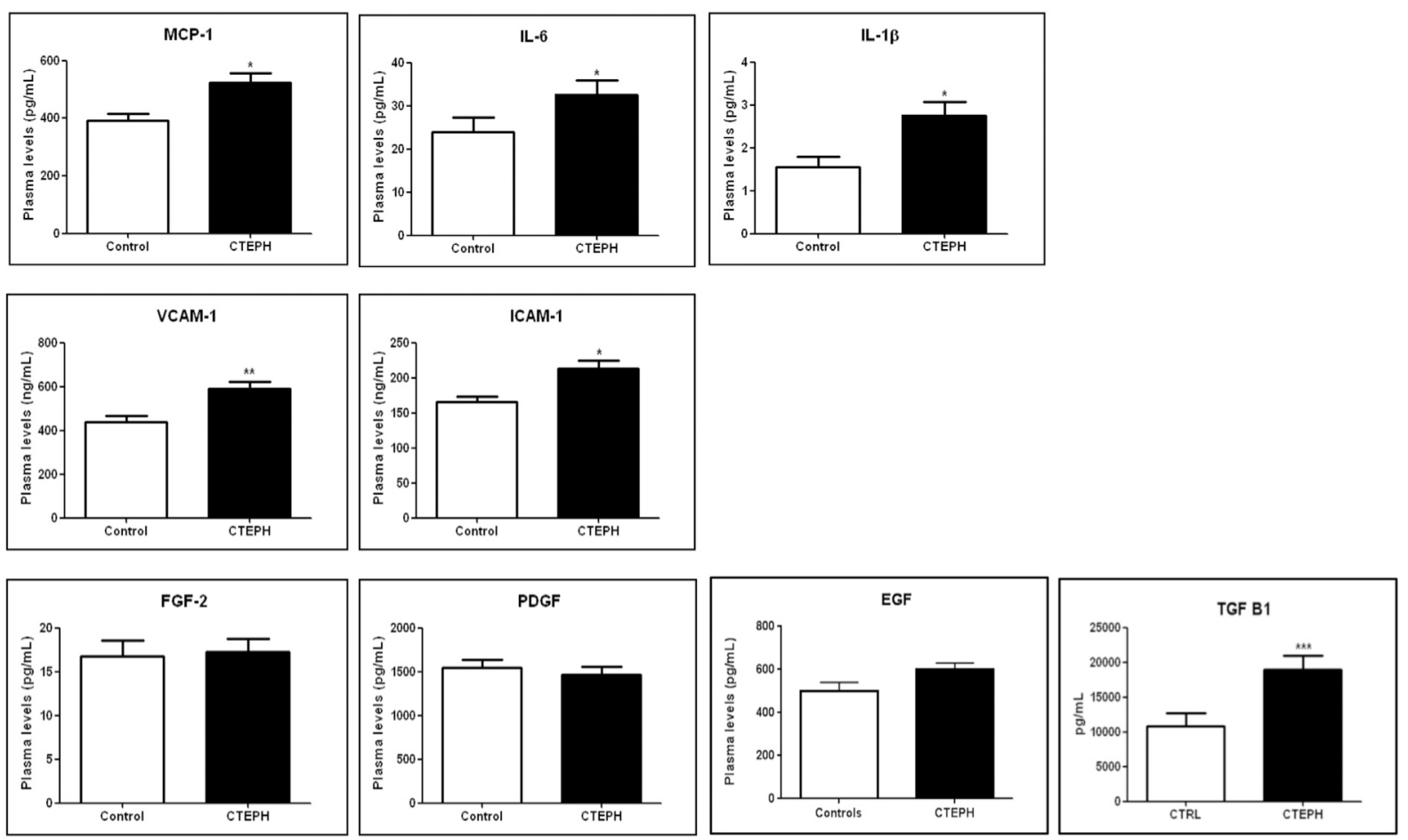

Figure 2 Plasma levels are shown for cytokines, adhesion molecules, and growth factors in 94 control patients and in 109 chronic thromboembolic pulmonary hypertension (CTEPH) patients. (A) Plasma levels of inflammatory cytokines monocyte chemoattractant protein1 (MCP-1), interleukin (IL) -6 , and IL-1 $\beta$ were significantly higher in CTEPH patients vs control patients. (B) Circulating levels of the cellular adhesion molecules vascular cell adhesion molecule 1 (VCAM-1) and intercellular adhesion molecule-1 (ICAM-1) were also significantly increased in CTEPH patients compared with the controls. (C) There was no difference between control and CTEPH patients in the plasma levels of the growth factors fibroblast growth factor (FGF-2), platelet-derived growth factor (PDGF), or endothelial growth factor (EGF). TGF- $\beta 1$, transforming growth factor $\beta 1$. Data are expressed as mean \pm standard error of the mean. $* p<0.05$, **p $<0.01$.

CTEPH patients had similar plasma concentrations of growth factors FGF-2 (16.74 \pm 7 vs $17.25 \pm 9 \mathrm{ng} / \mathrm{ml}, p$ $=0.914)$, PDGF $(1,469.81 \pm 45$ vs $1,545.51 \pm 27 \mathrm{ng} / \mathrm{ml}, p$ $=0.097)$, and $\operatorname{EGF}(0.51 \pm 0.03$ vs $0.60 \pm 0.20, p=$ $0.117)$. However, the plasma concentration of TGF- $\beta 1$ was significantly higher in CTEPH patients than in controls $(18,938 \pm 45$ vs $10,801 \pm 27 \mathrm{ng} / \mathrm{ml}, p=0.0001$ Figure 2C). Increased protein levels did not correlate with disease severity (data not shown).

\section{CTEPH-PEC medium increases PASMC proliferation}

There was no difference in the proliferative response of PASMC from CTEPH vs control patients when exposed to $5 \%$ FCS $(160 \pm 12$ vs $150 \pm 17 \mathrm{cpm} /$ well, $p=0.732$; and $1,286 \pm 50$ vs $1,200 \pm 40 \mathrm{cpm} /$ well, $p=0.825$; Figure $3 \mathrm{~A}$ ). However, control and CTEPH PASMC showed a statistically significant increase in growth when exposed to medium from CTEPH patient PECs $(970 \pm 30$ vs $530 \pm$ $27 \mathrm{cpm} /$ well, $p=0.032$ ) compared with medium from control PECs $(1,020 \pm 50$ vs $580 \pm 36 \mathrm{cpm} / \mathrm{well}, p=$ 0.0241 ; Figure 3B).

\section{CTEPH-PEC medium stimulates monocyte migration}

The presence of CTEPH-PEC medium in the Transwell lower chamber induced increased migration of control monocytes from the upper to the lower chamber compared with control-PEC medium ( $47 \pm 7$ vs $15 \pm 4$ cell number/ field, $p=0.0086$; Figure 4A). CTEPH-PEC medium increased the migration of CTEPH monocytes compared with control-PEC medium $(49 \pm 5$ vs $18 \pm 3$ cell number/ field, $p=0.0059$; Figure 4B). This effect was significantly reduced by the presence of a MCP-1-blocking antibody. There was no difference in the migration of CTEPH and control monocytes when both were exposed to control PEC or CTEPH PEC medium with anti-MCP1 (Figure 4A and B).

\section{Factor production by CTEPH-PECs}

Compared with the culture media from control PECs $(n=$ 10 in each group), CTEPH-PEC culture media contained significantly increased levels of known growth factors, inflammatory cytokines, and ICAM-1. Specifically, levels of MCP-1, IL-6, and IL-1 $\beta$ were significantly increased in the CTEPH-PEC media compared with control PEC media $(3,537 \pm 50 \mathrm{vs} 1,038 \pm 35 \mathrm{pg} / \mathrm{ml}, p=0.0054 ; 921 \pm 28 \mathrm{vs}$ $192 \pm 13 \mathrm{pg} / \mathrm{ml}, p=0.0015$; and $12.7 \pm 0.9$ vs $1.15 \pm$ $0.3 \mathrm{pg} / \mathrm{ml}, p=0.00231$, respectively; Figure $5 \mathrm{~A}$ ), as was the level of ICAM-1 $(1,692 \pm 96$ vs $307 \pm 12 \mathrm{pg} / \mathrm{ml}, p=$ $0.00347)$. There was no difference in the level of VCAM-1 ( $3.84 \pm 0.70$ vs $3.65 \pm 0.60 \mathrm{pg} / \mathrm{ml}, p=0.687$; Figure $5 \mathrm{~B})$. FGF-2 was significantly elevated in the media from CTEPH PEC compared with controls $(13.2 \pm 0.40$ vs $2.30 \pm$ $0.3 \mathrm{mg} / \mathrm{ml}, p=0.0098$ ). However, there was no difference in the levels of PDGF, EGF, or TGF- $\beta 1(1.53 \pm 0.3$ vs 1.28 
A

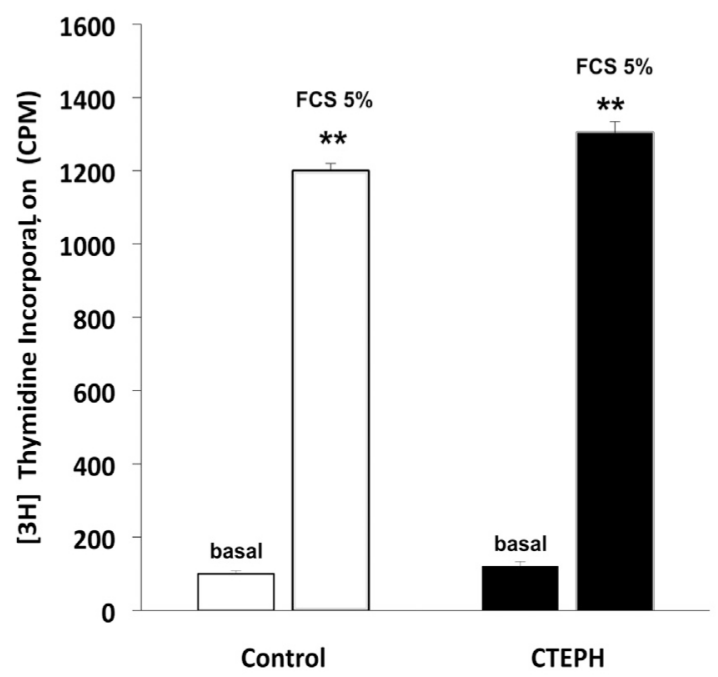

B

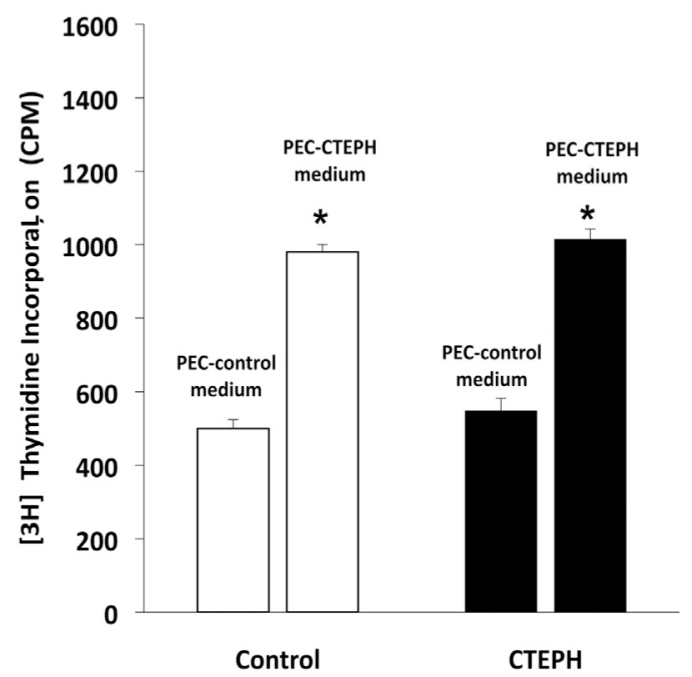

Figure 3 Exposure to chronic thromboembolic pulmonary hypertension (CTEPH) pulmonary endothelial cell (PEC) culture medium increases pulmonary artery smooth muscle cell (PASMC) growth. (A) As marked by incorporation of $\left[{ }^{3} \mathrm{H}\right]$ thymidine, there was no difference in the growth of control vs CTEPH pulmonary vascular SMCs at baseline (without treatment) or after exposure to 5\% fetal calf serum (FCS). (B) Control and CTEPH PASMCs both showed significantly increased growth, as marked by incorporation of $\left[{ }^{3} \mathrm{H}\right]$ thymidine, when exposed to culture media from CTEPH PEC than when exposed to culture media from control PEC. PASMC proliferation increased in response to CTEPH-PEC media regardless of the origin of the PASMC. Data are expressed as mean \pm standard error of the mean. $* p<0.05$, $* * p<0.01$.

$\pm 0.5 \mathrm{pg} / \mathrm{ml}, p=0.0925 ; 4.0 \pm 0.3$ vs $3.7 \pm 0.5 \mathrm{pg} / \mathrm{ml}, p=$ 0.879 ; and $152 \pm 45$ vs $220 \pm 27 \mathrm{ng} / \mathrm{ml}, p=0.1143$, respectively; Figure 5C).

\section{PEA specimen composition and structure}

Representative images of the gross and microscopic appearance of surgical specimens obtained from CTEPH patients, and their composition, are shown in Figure 6. The ratio of fibrous (organized thrombus) to fibrinous (unorganized thrombus) areas was 74\%:26\% (Figure 6B). Organized thrombus typically contained fibroblasts/myofibroblasts, inflammatory cells (mainly lymphocytes and

\section{Control monocytes}

A

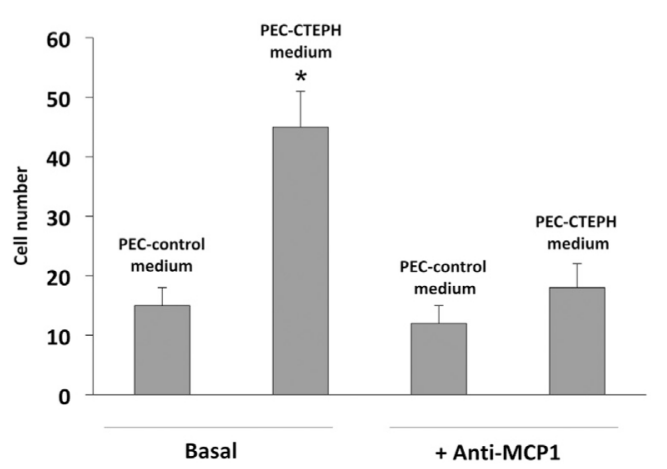

macrophages), and numerous neovessels of varying size (repermeabilization vessels).

\section{Discussion}

In this study, we show that PECs contribute to an inflammatory state in patients with CTEPH and that the increased production of inflammatory cytokines and growth factor FGF-2 by PECs stimulates the growth of PASMCs and increases monocyte migration. This large study examined PECs from CTEPH patients and shows how changes in the pulmonary endothelium can affect other key cells. These findings underline the importance of inflamma-

\section{CTEPH monocytes}

\section{B}

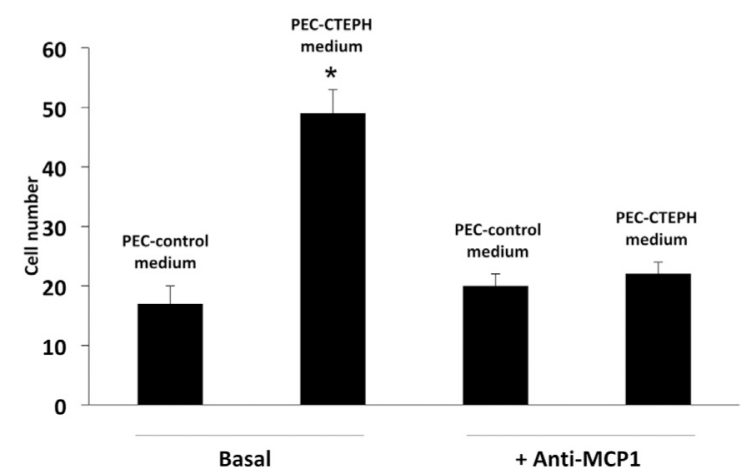

Figure 4 Effect of chronic thromboembolic pulmonary hypertension (CTEPH) pulmonary endothelial cell (PEC) culture media on monocyte migration. Exposure to CTEPH-PEC culture media significantly increased the migration of both (A) control and (B) CTEPH monocytes compared with culture media from control PEC. This effect was completely abolished in the presence of an anti-monocyte chemoattractant protein $1(\mathrm{MCP}-1)$ antibody. Data are expressed as mean of cells per field \pm standard error of the mean. $* p<0.01$. 
MCP-1

A

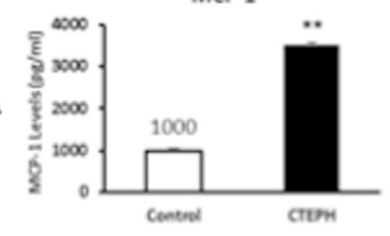

B

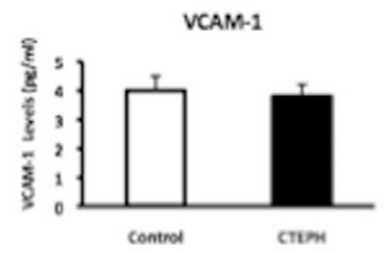

FGF-2

C

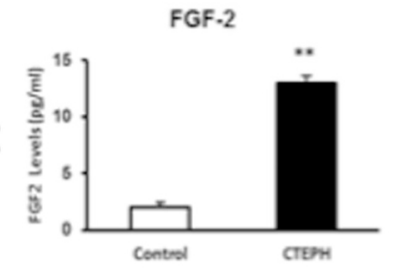

IL-6

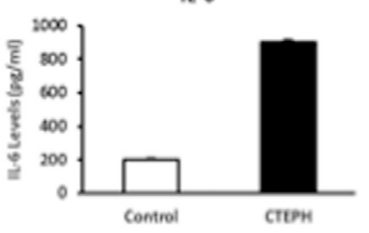

ICAM-1
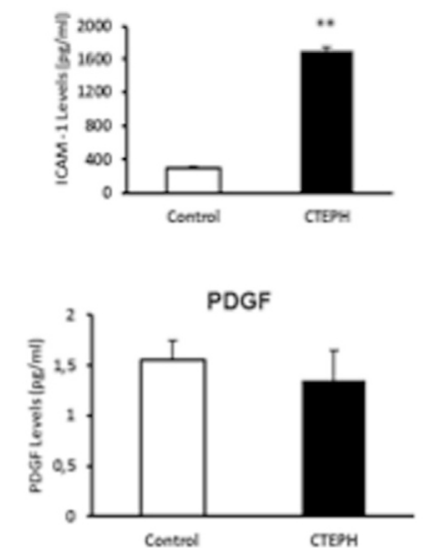

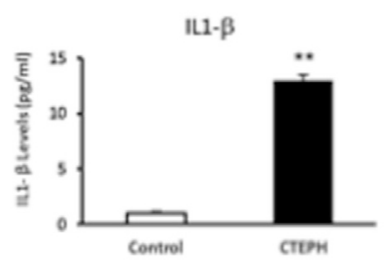

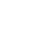
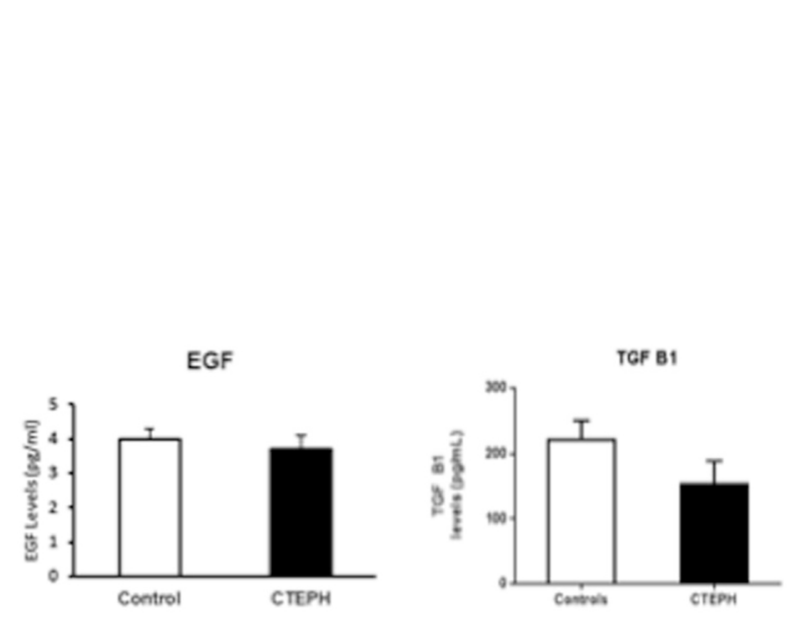

Figure 5 Chronic thromboembolic pulmonary hypertension (CTEPH) pulmonary endothelial cells (PECs) show increased production of inflammatory cytokines, adhesion molecules, and growth factors in their culture media. (A) The levels of inflammatory cytokines monocyte chemoattractant protein 1 (MCP-1), interleukin (IL) 6, and IL-1 $\beta$ were significantly increased in the culture media of CTEPH PECs compared with controls. (B) The level of intercellular adhesion molecule 1 (ICAM-1) was higher in culture media from CTEPH PECs than from control PECs. There was no difference in the level of vascular cellular adhesion molecule 1 (VCAM-1). (C) The level of fibroblast growth factor 2 (FGF-2) was significantly higher in CTEPH-PEC culture media than in control-PEC culture media. There was no difference in the levels of platelet-derived growth factor (PDGF) or epidermal growth factor (EGF). TGF- $\beta 1$, transforming growth factor- $\beta 1$. Data are expressed as mean \pm standard error of the mean. $* p<0.05$, $* * p<0.01$.

tion in the overall pathogenesis of CTEPH and suggest a potential mechanism for the increased cellular proliferation and inflammatory infiltration seen in PEA specimens.

One of the fundamental questions in CTEPH is how the presence of unresolved thrombi within the pulmonary arteries leads to the chronic clot and vessel fibrosis seen in the disease. Consequently, this study focuses on the pathophysiology underlying the progression from clot to fibrosis, with our hypothesis being that changes in the pulmonary artery endothelium contribute to the pathogenic vascular remodeling seen in CTEPH. Although inflammatory cytokines have been associated with other pathologic aspects of CTEPH, specifically microvascular disease and right ventricular dysfunction, these additional disease characteristics were not examined in this study. ${ }^{11,12}$

Patients with CTEPH demonstrate significant pulmonary artery remodeling and proliferation of SMCs. We found increased growth of PASMCs exposed to culture medium from CTEPH PECs. This could be due to paracrine signaling via the increased levels of FGF-2 found in CTEPH-PEC culture medium, because FGF-2 levels were not higher in the plasma from CTEPH patients but were significantly elevated in the culture media. This effect is similar to that seen in idiopathic $\mathrm{PAH}$, where the enhanced expression or activity of a variety of growth factors, including FGF, PDGF, and EGF, has been to shown to stimulate smooth muscle proliferation and lead to obstructive pulmonary vascular remodeling. ${ }^{13-15}$ Although the contribution of endothelial cells to PASMC proliferation via growth factor synthesis has been studied in PAH, this work has identified a similar mechanism in CTEPH. ${ }^{9,14}$

Like FGF-2, MCP-1 has also been shown to induce SMC proliferation in $\mathrm{PAH} .{ }^{10}$ Furthermore, previous studies have shown that MCP-1 is predominantly expressed in the endothelium of CTEPH patients, suggesting that PECs could be the primary source of abnormal MCP-1 expression. ${ }^{16}$ We found MCP-1 levels that were significantly higher in the culture medium from CTEPH PECs than from control PECs, as well as significantly increased plasma levels of MCP-1 in CTEPH patients. This overexpression of MCP-1 would stimulate SMC proliferation and may contribute to pathogenic vascular remodeling.

In addition, MCP-1 is a strong chemoattractant for inflammatory cells and a stimulant for fibrosis. MCP-1 stimulates expression of genes for fibroblast collagen and TGF- $\beta 1$ and stimulates physiologic and pathologic inflammation, including that seen in atherosclerosis. ${ }^{17,18}$ Monocytes from control and CTEPH patients showed increased migration when exposed to the culture medium from CTEPH PECs, and this effect was markedly diminished after an anti-MCP-1 antibody-inhibited MCP-1. These findings suggest a central role for MCP-1 in attracting monocytes to the thrombus and vessel wall of CTEPH patients.

IL-6 is a potent pro-inflammatory cytokine previously shown to be increased in PAH patients and in CTEPH 


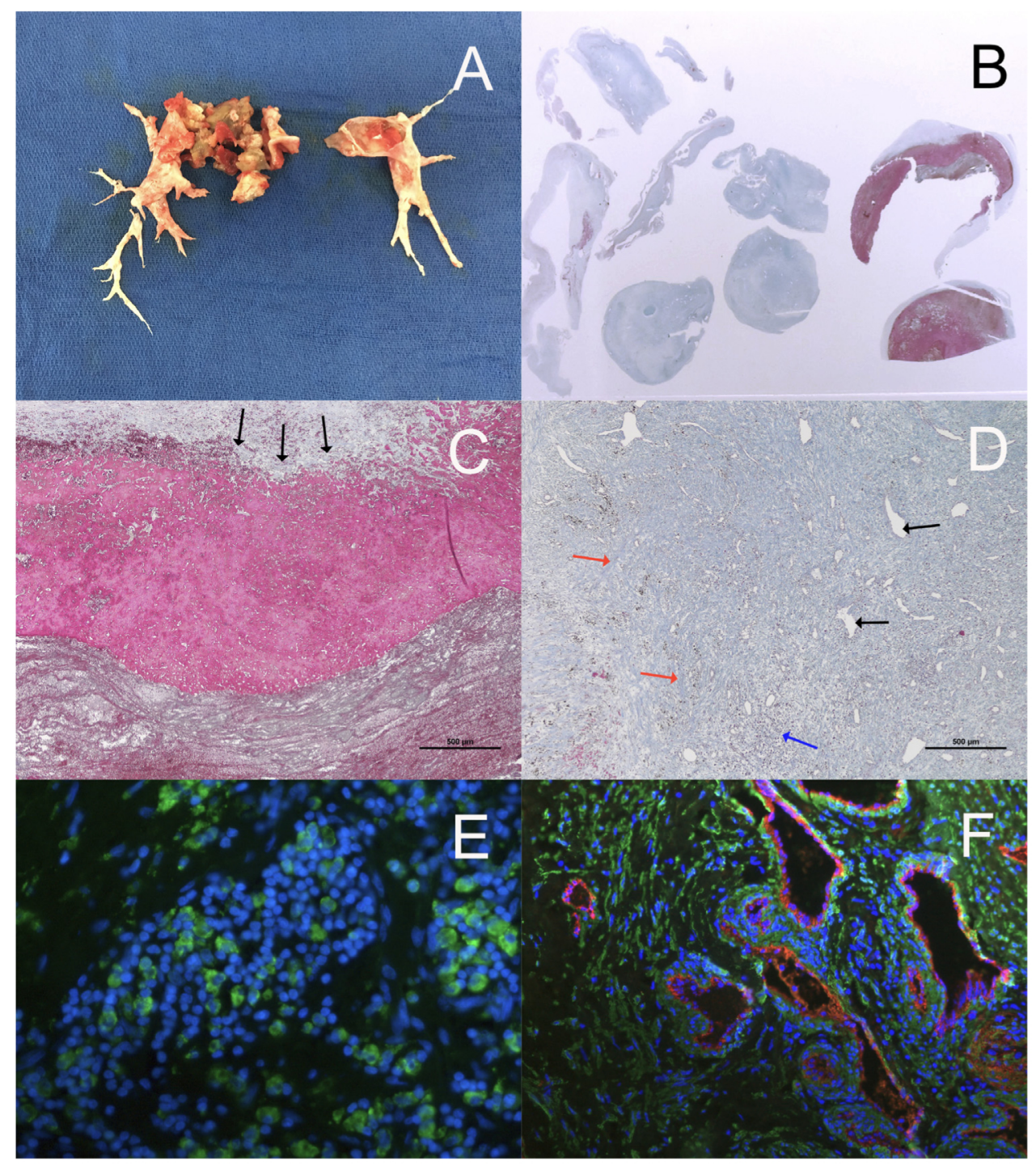

Figure 6 Pulmonary endarterectomy specimen with partially organized thromboembolic material. (A) Gross morphology of surgical specimen shows large, central, fresh thrombus with fibrous extension into segmental arteries. (B) Masson's trichrome staining of organized, collagen-rich material (blue) and recent, non-organized fibrin-rich material (red). The organized portion was estimated at 65\% (original magnification $\times 10$ ). (C) Fibrinous, fresh thrombotic portion (red), with peripheral organization by fibroblasts/myofibroblasts (arrows). The red-brownish material (bottom) corresponds to the most recent deposit, consisting of erythrocytes and platelets. (D) Organized, collagen-rich portion of the thrombus; the dense tissue is rich in fibroblasts/myofibroblasts (red arrows), accumulations of lymphocytes and other inflammatory cells (blue arrow), and numerous repermeabilization vessels (black arrows). Note the black pigment (top, left) corresponding to iron deposits, a sign of erythrocytic degradation. (E) Macrophages appear green after staining with anti-human cluster of differentiation (CD)-68 antibodies. (F) Staining with an anti-human von Willebrand factor antibody and an anti-human $\alpha$-actin antibody, respectively, demonstrates von Willebrand-positive endothelial cells (red) and $\alpha$-actin-positive smooth muscle cells (green). Nuclei were counterstained with $4^{\prime}$, 6'-diamino-2-phenylindole, and appear blue $(\mathrm{E}$ and $\mathrm{F})$. These images are at original magnification $\times 40$.

patients with isolated right ventricular dysfunction. ${ }^{12,19,20}$ We found increased IL-6 levels in culture medium from CTEPH PECs compared with controls, further supporting the contribution of PECs to the inflammatory state seen in CTEPH patients. The potential role for inflammation in the pathophysiology of CTEPH has been widely proposed, and increased inflammation in CTEPH patients is not just localized to the lung but systemically manifested with increased rates of inflammatory bowel disease and higher serum levels of C-reactive protein. ${ }^{21,22}$ The higher levels of IL-6 and IL-1 found in the culture medium of CTEPH PECs suggest that PECs may stimulate this inflammatory state and are consistent with the higher plasma level of TGF- $\beta 1$ seen in CTEPH patients despite the normal TGF- $\beta 1$ level in CTEPH PEC culture medium.

Taken in combination, increased levels of growth factors (FGF-2), pro-inflammatory cytokines (IL-1 and IL-6), and mediators of monocyte infiltration and fibrosis (MCP-1) in the medium of CTEPH PECs suggest that PECs play a central role in the pathogenesis of CTEPH. Furthermore, levels of VCAM-1 and ICAM-1 were elevated in the plasma of CTEPH patients and the level of ICAM-1 was elevated in the culture medium of CTEPH PECs. VCAM-1 and ICAM-1 promote monocyte adherence in response to pro-inflammatory signaling via IL-1 $\beta$ - and IL-6-induced C-reactive protein, and an increase in their expression is a 
marker of endothelial cell activation. ${ }^{23}$ PEC activation and dysfunction, as manifested by the aberrant expression of these proteins, could explain the fundamental pathologic progression from an unresolved thrombus to the chronic, maladaptive vascular remodeling seen in CTEPH.

Although we found increased levels of potentially pathologic molecules in CTEPH patient plasma, we did not find any correlation between these levels and the degree of the patients' PH. Notably, correlations between serum levels of IL- 6 and pulmonary vascular resistance and right atrial pressure, as well as MCP-1 and pulmonary vascular resistance, have been previously reported. ${ }^{16,24}$ This study examines a larger cohort of CTEPH patients than the previous studies, and the absence of a correlation may result from a greater diversity of patients. Furthermore, although mean pulmonary artery pressure and pulmonary vascular resistance both reflect disease severity and are closely related measures, they are different parameters. Because the severity of a patient's PH is likely multifactorial, it is also possible that a single cytokine, or small group of cytokines, does not entirely account for clinical status. Lastly, we examined a select sub-set of inflammatory cytokines, and this group may not include those that do correlate with disease severity.

Our study is largely descriptive, and therefore, it suggests a potential role for PECs in the pathophysiology of CTEPH but does not prove a causal relationship. In addition, there are several important differences between the control and CTEPH patient populations. Our control group consisted of patients undergoing lung resection for malignancy. Malignancy is a known inflammatory state, and this could have decreased the differences seen in inflammatory molecule levels. However, this is unlikely to have affected the overall findings.

Secondly, significantly more control patients used tobacco than CTEPH patients. Smoking is also known to induce inflammation and specifically to increase levels of IL- $6{ }^{25}$ Because this would increase the chance of a type II, rather than a type I, error, we believe it does not affect the validity of the differences found.

In conclusion, we report that CTEPH PECs express increased levels of inflammatory cytokines (IL1, IL6, MCP1), growth factor FGF, and adhesion molecule ICAM. These findings underscore the importance of inflammation in CTEPH, and specifically suggest that PEC dysfunction may be the mechanism underlying the progression of unresolved thrombus to fibrotic vascular remodeling. Further studies investigating the trigger of this inflammation, the role of the involved inflammatory, growth, or adhesion factors, and the potential role of monocytes in its initiation are needed to improve our understanding of the pathophysiology of this lethal disease.

\section{Disclosure statement}

None of the authors has a financial relationship with a commercial entity that has an interest in the subject of the presented manuscript or other conflicts of interest to disclose.
This work was funded by the Programme Hospitalier de Recherche Clinique National 2009 of the French Ministry of Health; RCB 2009-A0098057.

\section{References}

1. Dartevelle P, Fadel E, Mussot S, et al. Chronic thromboembolic pulmonary hypertension. Eur Respir J 2004;23:637-48.

2. Pengo V, Lensing AWA, Prins $\mathbf{M H}$, et al. Incidence of chronic thromboembolic pulmonary hypertension after pulmonary embolism. N Engl J Med 2004;350:2257-64.

3. Tapson VF, Humbert M. Incidence and prevalence of chronic thromboembolic pulmonary hypertension: from acute to chronic pulmonary embolism. Proc Am Thorac Soc 2006;3:564-7.

4. Pepke-Zaba J, Delcroix M, Lang I, et al. Chronic thromboembolic pulmonary hypertension (CTEPH) results from an international prospective registry. Circulation 2011;124:1973-81.

5. Humphries J, McGuinness CL, Smith A, Waltham M, Poston R, Burnand KG. Monocyte chemotactic protein-1 (MCP-1) accelerates the organization and resolution of venous thrombi. J Vasc Surg 1999;30: 894-900.

6. Londy FJ, Kadell AM, Wrobleski SK, Prince MR, Strieter RM, Wakefield TW. Detection of perivenous inflammation in a rat model of venous thrombosis using MRV. J Investig Surg Off J Acad Surg Res 1999; 12:151-6.

7. Henke PK, Varga A, De S, et al. Deep vein thrombosis resolution is modulated by monocyte CXCR2-mediated activity in a mouse model. Arterioscler Thromb Vasc Biol 2004;24:1130-7.

8. Wakefield TW, Strieter RM, Wilke CA, et al. Venous thrombosisassociated inflammation and attenuation with neutralizing antibodies to cytokines and adhesion molecules. Arterioscler Thromb Vasc Biol 1995; 15:258-258.

9. Eddahibi S, Guignabert C, Barlier-Mur AM, et al. Cross talk between endothelial and smooth muscle cells in pulmonary hypertension critical role for serotonin-induced smooth muscle hyperplasia. Circulation 2006;113:1857-64.

10. Sanchez O, Marcos E, Perros F, et al. Role of endothelium-derived CC chemokine ligand 2 in idiopathic pulmonary arterial hypertension. Am J Respir Crit Care Med 2007;176:1041-7.

11. Boulate D, Perros F, Dorfmuller P, et al. Pulmonary microvascular lesions regress in reperfused chronic thromboembolic pulmonary hypertension. J Heart Lung Transplant 2015;34:457-67.

12. Haehling $S$ von, Bardeleben RS, Kramm $T$ von, et al. Inflammation in right ventricular dysfunction due to thromboembolic pulmonary hypertension. Int J Cardiol 2010;144:206-11.

13. Perros F, Montani D, Dorfmüller P, et al. Platelet-derived growth factor expression and function in idiopathic pulmonary arterial hypertension. Am J Respir Crit Care Med 2008;178:81-8.

14. Izikki M, Guignabert C, Fadel E, et al. Endothelial-derived FGF2 contributes to the progression of pulmonary hypertension in humans and rodents. J Clin Invest 2009;119:512-23.

15. Merklinger SL, Jones PL, Martinez EC, Rabinovitch M. Epidermal growth factor receptor blockade mediates smooth muscle cell apoptosis and improves survival in rats with pulmonary hypertension. Circulation 2005;112:423-31.

16. Kimura H, Okada O, Tanabe $\mathrm{N}$, et al. Plasma monocyte chemoattractant protein-1 and pulmonary vascular resistance in chronic thromboembolic pulmonary hypertension. Am J Respir Crit Care Med 2001;164:319-24.

17. Gharaee-Kermani M, Denholm EM, Phan SH. Costimulation of fibroblast collagen and transforming growth factor $\beta 1$ gene expression by monocyte chemoattractant protein-1 via specific receptors. J Biol Chem 1996;271:17779-84.

18. Liptay MJ, Parks WC, Mecham RP, et al. Neointimal macrophages colocalize with extracellular matrix gene expression in human atherosclerotic pulmonary arteries. J Clin Invest 1993;91:588-94.

19. Soon E, Holmes AM, Treacy CM, et al. Elevated levels of inflammatory cytokines predict survival in idiopathic and familial pulmonary arterial hypertension. Circulation 2010;122:920-7. 
20. Humbert M, Monti G, Brenot F, et al. Increased interleukin-1 and interleukin-6 serum concentrations in severe primary pulmonary hypertension. Am J Respir Crit Care Med 1995;151:1628-31.

21. Bonderman D, Wilkens H, Wakounig S, et al. Risk factors for chronic thromboembolic pulmonary hypertension. Eur Respir J 2009;33: 325-331.

22. Quarck R, Nawrot T, Meyns B, Delcroix M. C-reactive protein: a new predictor of adverse outcome in pulmonary arterial hypertension. $\mathbf{J} \mathrm{Am}$ Coll Cardiol 2009;53:1211-8.
23. Szmitko PE. New markers of inflammation and endothelial cell activation: part I. Circulation 2003;108:1917-23.

24. Zabini D, Heinemann A, Foris V, et al. Comprehensive analysis of inflammatory markers in chronic thromboembolic pulmonary hypertension patients. Eur Respir J 2014;44:951-62.

25. McEvoy JW, Nasir K, DeFilippis AP, et al. Relationship of cigarette smoking with inflammation and subclinical vascular disease the MultiEthnic Study of Atherosclerosis. Arterioscler Thromb Vasc Biol 2015;35:1002-10. 